\title{
Synthesis, Properties, and Photovoltaic Performance of a Donor-Acceptor Copolymer Having Pyradinobisthiazole as the Acceptor Unit
}

\author{
Yutaka Ie*, Shohei Sasada, Makoto Karakawa, and Yoshio Aso* \\ The Institute of Scientific and Industrial Research (ISIR), Osaka University, \\ 8-1, Mihogaoka, Ibaraki, Osaka 567-0047, Japan
}

Keywords: pyradinobisthiazole, electron-deficient unit, donor-acceptor copolymer, organic semiconductor, organic photovoltaics

\section{Introduction}

The development of donor-acceptor (D-A) copolymers, which consist of alternating electron-rich and electron-deficient units, has become subjects of intense interest, since it offers the straightforward approaches as hole-transporting (p-type) semiconducting materials in organic photovoltaics (OPVs) in terms of fulfilling a reduced band gap to broaden light absorption to the long-wavelength region and a low-lying highest occupied molecular orbital (HOMO) energy level to increase the open-circuit voltage [1,2]. Although a variety of $\pi$-conjugated compounds including benzo[1,2-b:4,5- $\left.b^{\prime}\right]$ dithiophene (BDT) have been developed as donor units in D-A copolymers, the effective acceptor units are still limited due to the difficulty of molecular design that fulfills lowering the lowest unoccupied molecular orbital (LUMO). Thiazole-fused aromatic compounds such as thiazolothiazole and benzobisthiazole (BBTz) have been utilized as typical acceptor units in semiconducting materials [3-8]. On the other hand, we have recently reported that pyradinobisthiazole (PBTz), which replaced the benzene ring in BBTz with an electron-deficient pyrazine ring, can function as an acceptor unit in both p-type D-A and n-type A-A copolymers [9]. However, the potential of the PBTz unit under the combination with a typical donor unit is still unclear. In this paper, we report the synthesis, properties, and photovoltaic performance of a new D-A copolymer P-PBTz-T ${ }^{\mathbf{1 2}}$-BDT based on BDT and PBTz as donor and acceptor units, respectively (Fig. 1).

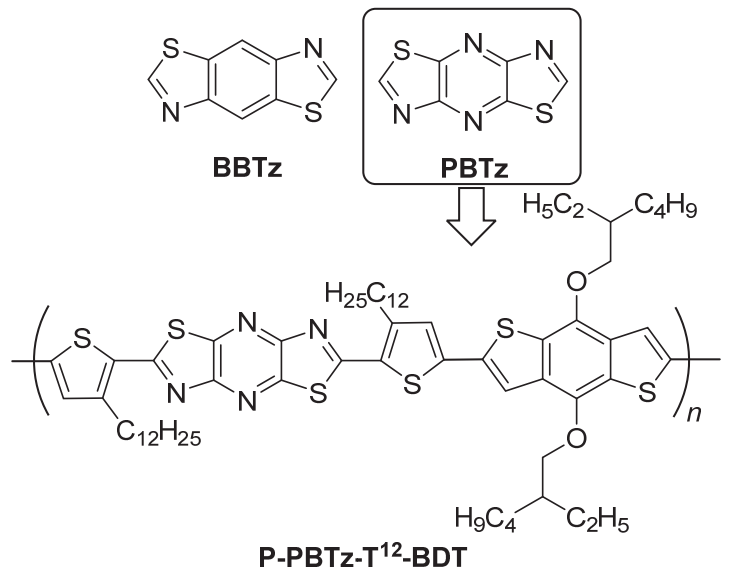

Fig. 1. Chemical structures of BBTz, PBTz, P-PBTz-T ${ }^{12}$-BDT.

\section{Experimental}

Microwave irradiation was performed by a Biotage Initiator Ver. 2.5. The microwave power output was set at $400 \mathrm{~W}$. The reaction temperature was kept at $180{ }^{\circ} \mathrm{C}$, and internal temperature during the reaction was monitored by IR sensor. Analytical gel-permeation chromatography (GPC) was performed on a Hitachi High-Technologies Corporation L-2420/L-2130 equipped with a Shodex K-803L at $40{ }^{\circ} \mathrm{C}$ using chloroform as eluent. UV-vis spectra were recorded on a Shimadzu UV-3600 spectrophotometer. Photoelectron spectroscopy in air (PESA) was carried out using a Riken Keiki Co. Ltd. AC-3 with a light intensity of $10 \mathrm{~mW}$.

Indium tin oxide (ITO)-coated glass substrates were first cleaned by ultrasonication in toluene, acetone, $\mathrm{H}_{2} \mathrm{O}$, and 2-propanol for $10 \mathrm{~min}$, 
respectively, followed by $\mathrm{O}_{2}$ plasma treatment for 10 min. $\mathrm{ZnO}$ layer was fabricated by spin-coating of a solution consists of $\mathrm{Zn}(\mathrm{OAc})_{2} \cdot 2 \mathrm{H}_{2} \mathrm{O} \quad(500 \mathrm{mg})$, 2-aminoethanol $(144 \mathrm{mg})$ and 2-methoxyethanol (5 $\mathrm{mL}$ ) on the ITO surface at $3000 \mathrm{rpm}$ for $30 \mathrm{sec}$, followed by a thermal treatment at $200{ }^{\circ} \mathrm{C}$ for $30 \mathrm{~min}$ in ambient conditions. The active layers were then prepared by spin-coating on the ITO/ZnO electrode at $1000 \mathrm{rpm}$ for $1 \mathrm{~min}$ in a glove box. The typical thickness of the active layer was $70-90 \mathrm{~nm}$. Finally, $\mathrm{MoO}_{3}$ and $\mathrm{Ag}$ electrodes were evaporated on the top of active layer through a shadow mask to define the active area of the devices $\left(0.09 \mathrm{~cm}^{2}\right)$ under a vacuum of $10^{-5} \mathrm{~Pa}$ to a thickness of 10 and $100 \mathrm{~nm}$ determined by a quartz crystal monitor. After sealing the device from the air, the photovoltaic characteristics were measured in air under simulated air mass 1.5 global (AM $1.5 \mathrm{G}$ ) with an irradiation intensity of $100 \mathrm{~mW} \mathrm{~cm}{ }^{-2}$ (SAN-EI ELECTRIC, XES-301S). The current density-voltage characteristics of photovoltaic devices were measured by using a KEITHLEY 2400 source meter. The EQE spectra were measured by using a Soma Optics Ltd. S-9240.

\section{Results and discussion}

The monomer unit $\mathbf{1}$ was prepared by our established synthetic route [9]. Then, 1 was copolymerized with 1,1'-[4,8-bis[(2-ethylhexyl)oxy]benzo[1,2-b:4,5-b'] dithiophene-2,6-diyl]bis(1,1,1-trimethylstannane)

(2) by the microwave-assisted Stille coupling reaction in the presence of $\mathrm{Pd}_{2}(\mathrm{dba})_{3} \cdot \mathrm{CHCl}_{3}$ and $\mathrm{P}(o \text {-tolyl })_{3}$ in toluene/DMF $(5 / 1)$ to give P-PBTz-T ${ }^{12}$-BDT in $86 \%$ yield (Scheme 1). The synthesized copolymer was purified using a Soxhlet apparatus by successive washing with methanol and hexane to remove low-molecular-weight fractions, and finally by extracting with chloroform. Analytical GPC using a polystyrene standard determined the number-average molecular weight of P-PBTz-T ${ }^{12}$-BDT to be $15.9 \mathrm{kDa}$ with molecular-weight dispersity of 3.2 .

The thermal gravimetrical analysis (TGA) indicated that this copolymer possesses high thermal stability with $5 \%$-weight-loss temperature of $294{ }^{\circ} \mathrm{C}$ (Fig. 2).

The photophysical properties in a chloroform solution and thin films were measured by UV-vis absorption spectrometry (Fig. 3). In chloroform solution, P-PBTz-T ${ }^{\mathbf{1 2}}$-BDT showed the absorption maximum at $552 \mathrm{~nm}$. The absorption spectrum in a thin film showed apparent broadening between 400 and $500 \mathrm{~nm}$ with a distinct shoulder at $630 \mathrm{~nm}$. This phenomenon in the solid state is generally regarded as the formation of crystalline structure. However, X-ray diffraction (XRD) measurements of the copolymer pristine films did not show any diffraction pattern (Fig. 4), indicating the amorphous nature in the solid state. The optical HOMO-LUMO energy gap $\left(\Delta E_{\mathrm{g}}{ }^{\mathrm{opt}}\right)$ extracted from the absorption onset of the film was $1.86 \mathrm{eV}$.

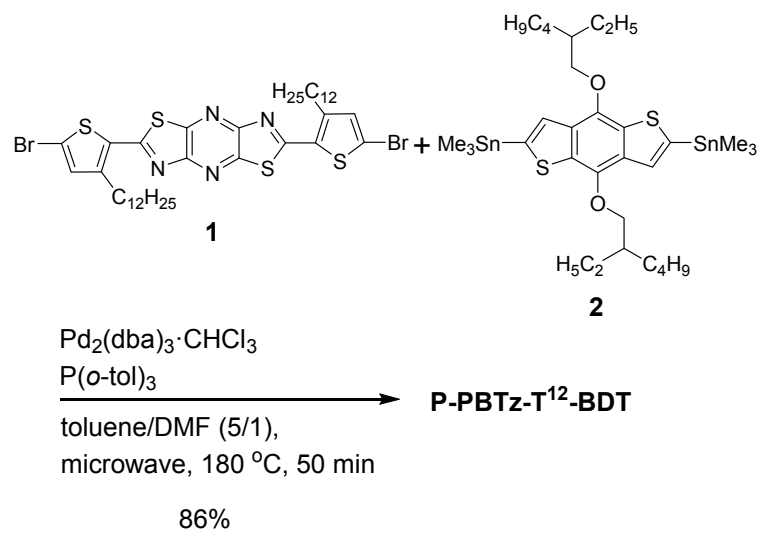

Scheme 1. Copolymerization of P-PBTz-T' ${ }^{12}$-BDT.

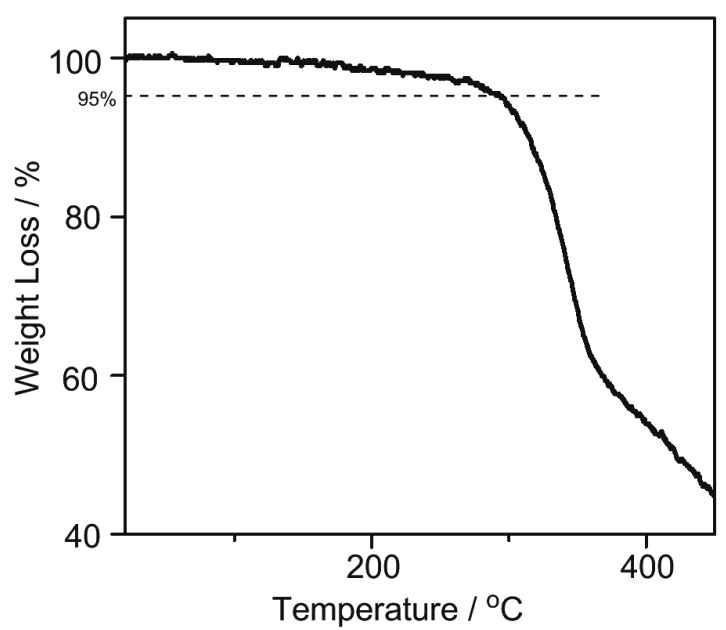

Fig. 2. TGA curve of P-PBTz-T ${ }^{\mathbf{1 2}}$-BDT with a scanning rate of $10^{\circ} \mathrm{C} \min ^{-1}$ under $\mathrm{N}_{2}$.

This value is smaller than the corresponding copolymer consisting of BDT and BBTz $(2.13 \mathrm{eV})$ [5], On the basis of similar HOMO energy levels of these copolymers in the electrochemical estimation ( $-5.71 \mathrm{eV}$ for BBTz-based coplymer [5] and -5.65 $\mathrm{eV}$ for PBTz-based copolymer), the smaller $\Delta E_{\mathrm{g}}{ }^{\text {opt }}$ value of P-PBTz-T $\mathbf{T}^{\mathbf{1 2}}$-BDT than that of the BBTz-based copolymer indicates the lower LUMO energy level of P-PBTz-T ${ }^{\mathbf{1 2}}$-BDT and thus the more electron-deficient characteristics of the PBTz unit than that of BBTz. 


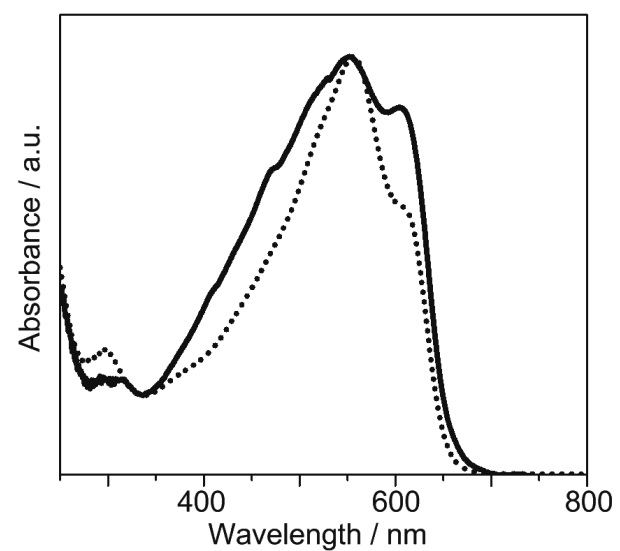

Fig. 3. UV-vis absorption spectra of P-PBTz-T ${ }^{\mathbf{1 2}}$-BDT in chloroform solution (dashed line) and as film (solid line).

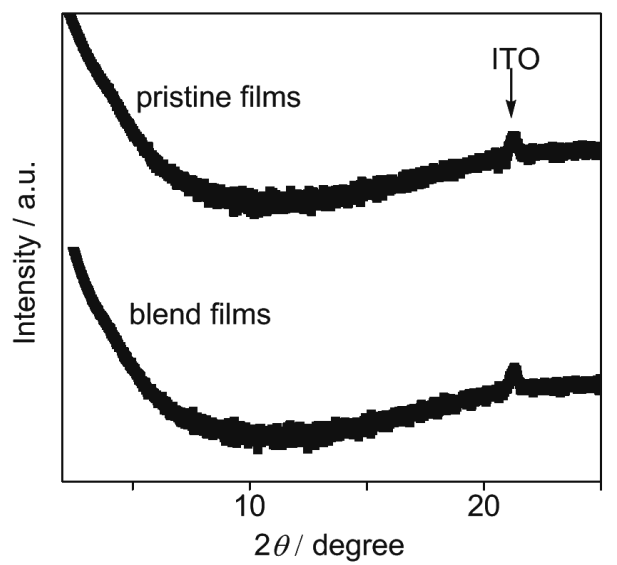

Fig. 4. XRD measurements of the pristine P-PBTz-T ${ }^{12}$-BDT and P-PBTz-T ${ }^{12}$-BDT/PC ${ }_{61} \mathrm{BM}$ blend films on $\mathrm{ZnO}$-coated ITO glass.

Further accurately, the ionization potential of the thin film was measured using PESA, which determined that the HOMO energy level $\left(E_{\text {НОмо }}\right)$ of P-PBTz-T ${ }^{12}$-BDT is $-5.51 \mathrm{eV}$ (Fig. 5). Based on $E_{\mathrm{HOMO}}$ and $\Delta E_{\mathrm{g}}$ opt,$E_{\mathrm{LUMO}}$ was calculated to be $-3.65 \mathrm{eV}$.

The charge-transporting characteristics of P-PBTz-T ${ }^{12}$-BDT was investigated by the space-charge-limited currents (SCLC) method. Hole-only device was prepared with the structure of ITO/PEDOT:PSS/P-PBTz-T ${ }^{\mathbf{1 2}}$-BDT/Au. The active layer was prepared from $10 \mathrm{mg} \mathrm{mL}^{-1}$ chloroform solution of the copolymer. The hole mobility of this device was calculated by the following equation: $J=9 V^{2} \varepsilon \varepsilon_{0} \mu / 8 d^{3}$, where $\varepsilon, \varepsilon_{0}, \mu$, and $d$ are the dielectric constant of the active layer, the permittivity of free space, the carrier mobility, and the measured thickness of the active layer, respectively. Under this condition, P-PBTz-T ${ }^{\mathbf{1 2}}$-BDT showed hole mobility of $4.3 \times$ $10^{-6} \mathrm{~cm}^{2} \mathrm{~V}^{-1} \mathrm{~s}^{-1}$ (Fig. 6), whose value is almost comparable to that of our developed PBTz-containing p-type copolymer [9]. The appearance of hole-transporting characteristics indicates that this copolymer has a potential for p-type OPV semiconducting material.

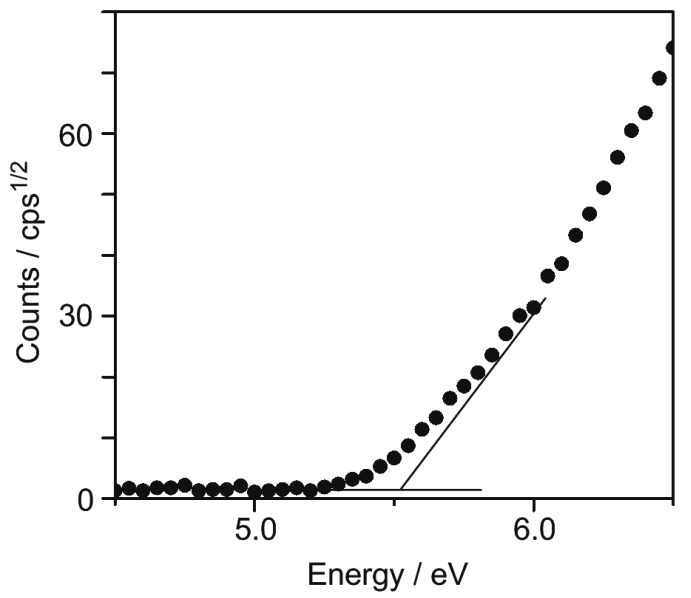

Fig. 5. PESA of P-PBTz-T ${ }^{12}$-BDT film.

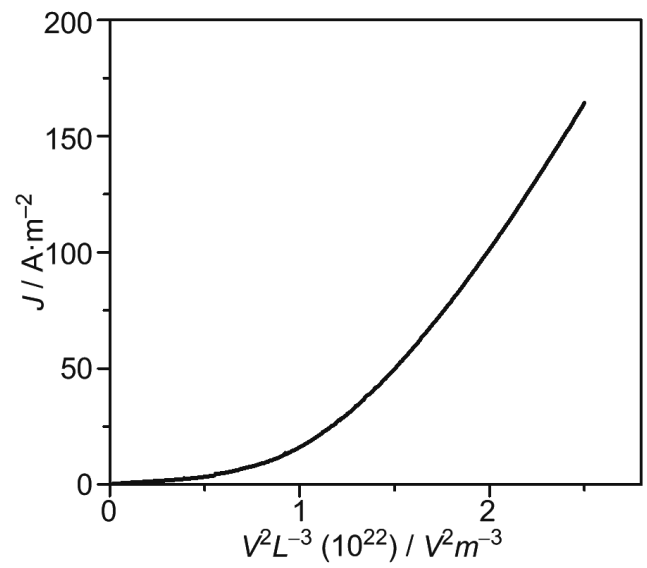

Fig. 6. $J-V$ characteristics of hole-only device for P-PBTz-T ${ }^{12}$-BDT.

In order to evaluate the OPV performance of P-PBTz-T ${ }^{12}$-BDT, bulk hetero-junction (BHJ) solar cell was fabricated with an inverted structure of glass/ITO/ZnO/active layer/MoOx/Ag. To investigate the p-type characteristics of P-PBTz-T ${ }^{12}$-BDT, the active layer was fabricated under the combination of P-PBTz-T ${ }^{\mathbf{1 2}}$-BDT with $[6,6]$-phenyl- $\mathrm{C}_{61}$-butyric acid methyl ester $\left(\mathrm{PC}_{61} \mathrm{BM}\right)$ as an n-type semiconductor. The fabrication conditions of the active layer were optimized by varying the blending ratio, concentration, and processing solvent; the optimal weight ratio of $\mathbf{P}-\mathbf{P B T z}-\mathbf{T}^{\mathbf{1 2}}$-BDT/ $/ \mathrm{PC}_{61} \mathrm{BM}$ combinations was $1: 1,10 \mathrm{mg} \mathrm{mL}^{-1}$ of $o$-dichlorobenzene/chloroform (4/1) solution. The active layer was prepared by spin-coating under a nitrogen atmosphere without thermal annealing. This device showed typical photovoltaic 
characteristics with a power conversion efficiency (PCE) of $1.08 \%$, with an open-circuit voltage of $0.79 \mathrm{~V}$, a short-circuit current density of $3.45 \mathrm{~mA}$ $\mathrm{cm}^{-2}$, and a fill factor of 0.40 (Fig. 7(a)). This PCE value is comparable to that for the reported OPV devices based on the corresponding BBTz-incorporated copolymers with $\mathrm{PC}_{71} \mathrm{BM}$ under similar fabricating conditions [5]. The external quantum efficiency (EQE) spectrum of this device exhibited photoresponses between 300 and $700 \mathrm{~nm}$ with a maximum value of $20 \%$ at 550 nm (Fig. 7(b)).
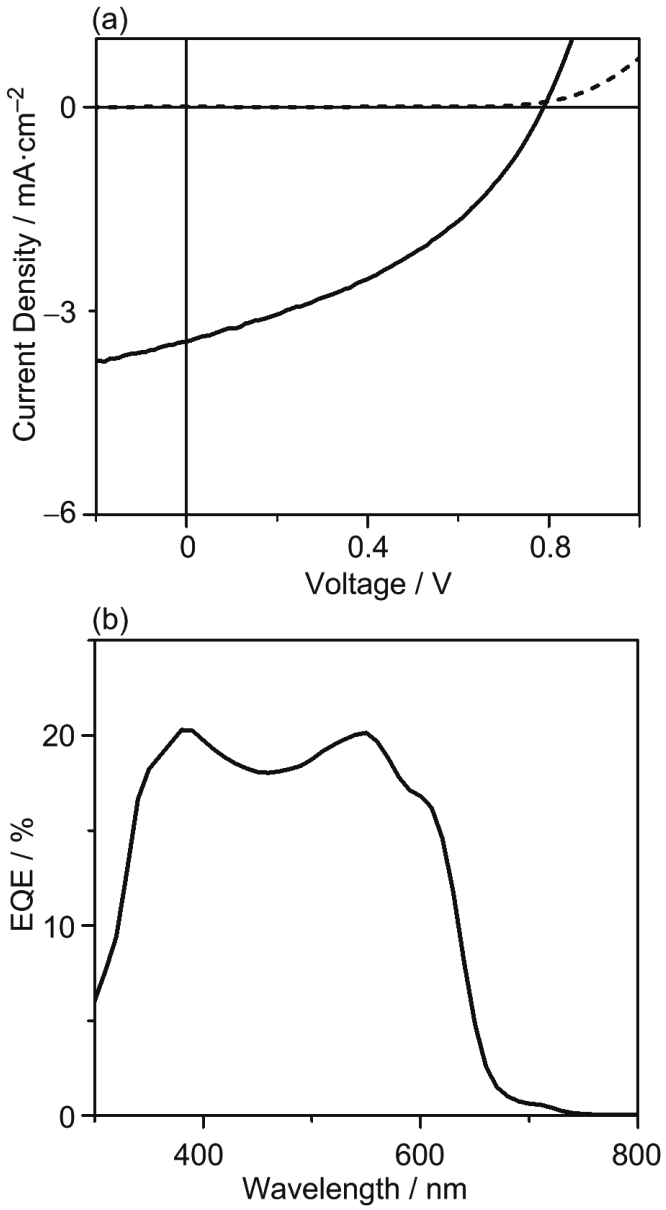

Fig. 7. (a) $J-V$ curves under illumination (solid line) and dark (dashed line) and (b) EQE spectrum of the

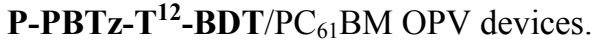

No characteristic crystallization peaks were detected by the XRD profile of the blend films (Fig. 4). Atomic force microscopy (AFM) images showed relatively smooth and featureless surface morphology (Fig. 8). These results indicate the amorphous nature of the blend film.

In summary, we developed PBTz-containing new D-A copolymer P-PBTz-T ${ }^{\mathbf{1 2}}$-BDT. $E_{\text {HOмо }}$ and $E_{\mathrm{LUMO}}$ were determined by the combination of PESA and UV-vis measurements in the solid state. SCLC measurements showed the hole-transporting characteristics. BHJ solar cells based on P-PBTz-T ${ }^{12}$-BDT and $\mathrm{PC}_{61} \mathrm{BM}$ showed a moderate PCE of $1.08 \%$. To further improve the PCE, the fine-tuning of the film morphology by the alkyl side-chain engineering and/or the structural modification of $\pi$-conjugated backbones have become important, which will lead to increasing the crystallinity in the solid state. Nevertheless, the obtained results confirm that PBTz has been positioned as a good candidate for the acceptor unit for the development of high-performance organic semiconducting materials.

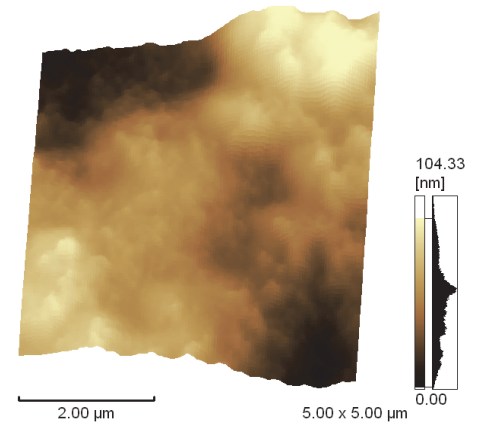

Fig. 8. AFM image of the P-PBTz-T ${ }^{\mathbf{1 2}}$-BDT/PC ${ }_{61} \mathrm{BM}$ blend films.

This work was supported by JST-PRESTO and MEXT, Japan. This work was partially supported by the Collaborative Research Program of the Institute for Chemical Research in Kyoto University with Prof. Yasujiro Murata and Dr. Atsushi Wakamiya (grant 2016-37).

\section{References}

1. H. Zhou, L. Yang, W. You, Macromolecules, 45 (2012) 607.

2. Z.-G. Zhang, J. Wang, J. Mater. Chem., 22 (2012) 4178

3. J. F. Wolfe, B. H. Loo, F. E. Arnold, Macromolecules, 14 (1981) 915.

4. J. A. Osaheni, S. A. Jenekhe, Chem. Mater., 4 (1992) 1282.

5. E. Ahmed, S. Subramaniyan, F. S. Kim, H. Xin, S. A. Jenekhe, Macromolecules, 14 (2011) 7207.

6. M. Mamada, J.-i. Nishida, S. Tokito, Y. Yamashita, Chem. Lett., 37 (2008) 766.

7. I. Osaka, K. Takimiya, R. D. McCullough, $A d v$. Mater, 22 (2010), 4993.

8. J. F. Mike, J. J. Intemann, M. Cai, T. Xiao, R. Shinar, J. Shinar, M. Jeffries-EL, Polym. Chem., 2 (2011) 2299.

9. Y. Ie, S. Sasada, M. Karakawa, Y. Aso, Org. Lett., 17 (2015) 4580. 